\title{
Links between Musicality and Vocal Emotion Perception
}

\author{
Christine Nussbaum and Stefan R. Schweinberger
}

Department for General Psychology and Cognitive Neuroscience, Friedrich Schiller University Jena, Germany

Correspondence should be addressed to Christine Nussbaum, Department for General Psychology and Cognitive Neuroscience, Friedrich Schiller University Jena, Leutragraben 1, 07743 Jena, Germany. Tel: +49 (0) 3641 945939, E-Mail: christine.nussbaum@uni-jena.de; or to Stefan R. Schweinberger, Department for General Psychology and Cognitive Neuroscience, Friedrich Schiller University Jena, Am Steiger 3/Haus 1, 07743 Jena, Germany. Tel: +49 (0) 3641 945181, E-Mail: stefan.schweinberger@uni-jena.de.

Note: This manuscript has not yet been peer-reviewed. The paper has been submitted on September 24, 2020, to Emotion Review, and is currently being under review there. 


\section{Abstract}

Links between musicality and vocal emotion perception skills have only recently emerged as a focus of study. Here we review current evidence for or against such links. Based on a systematic literature search, we identified 33 studies that addressed either (1) vocal emotion perception in musicians and non-musicians, (2) effects of musical training intervention on vocal emotion perception, (3) potentials of musical training for cochlear implant users, (4) vocal emotion perception in individuals with congenital amusia, or (5) the role of individual differences (e.g., musical interests, psychoacoustic abilities). Overall, the evidence supports a link between musicality and vocal emotion perception abilities. We discuss potential underlying factors, including origins of musicality in nature and nurture.

\section{Keywords}

Vocal emotion perception, musicality, musical training, amusia 


\section{Introduction and Review of Related Literature}

Human social communication depends on the exchange and mutual representation of multiple social signals. Among these, vocally expressed emotions are fundamental to human interaction (Grandjean, 2020; Sauter, 2017; Scherer, 1986; Scherer et al., 2016; A. W. Young et al., 2020). For several decades, this field was dominated by the view that humans perceive emotions efficiently and automatically. More recently however, prominent interindividual differences in vocal emotion perception skills became a focus of attention (Mill et al., 2009; Schirmer et al., 2005). For humans, voices and music are both prominent means of auditory communication of emotions. Some researchers have emphasized the similarities in the acoustic features of certain emotions in the voice and in music (Juslin \& Laukka, 2003; Scherer, 1995), whereas others reported similarities in the neural circuits involved in recognizing basic emotions from music as compared to voices (Aubé et al., 2015). Accordingly, differences in vocal emotion perception skills could be associated with different levels of musicality. Music forms a central part of human culture, and appreciation of music is interconnected with intense emotional experiences (Schäfer et al., 2013). However, there is huge variation in terms of musical aptitude and musical training. Here we integrate the currently available research that assessed possible links between musicality - in terms of both aptitude and training effects - and vocal emotion perception.

This review adds vocal emotion perception to several previous integrative works that discussed the association of musical training with other non-musical abilities. Compared to non-musical peers, musicians show superior pitch, temporal and timbre processing (Kraus \& Chandrasekaran, 2010) and those advantages expand from the musical into the vocal domain (Chartrand et al., 2008). Beyond the auditory modality, musicians have better audiovisual and auditory-motor integration, working memory, spatial abilities, executive functioning, general intelligence as well as speech and language skills (Elmer et al., 2018; Schellenberg, 2001, 2016). At the brain level, musicality is associated with widespread functional and structural differences, such as larger grey matter volume and stronger 
connectivity between areas associated with auditory, motor and visuospatial functions (Kraus \& Chandrasekaran, 2010; Pantev \& Herholz, 2011). Against this background of established links between musicality and comparitively distant competencies, the field remarkably lacks systematic integration of evidence concerning the related domain of voices, and vocal emotion perception in particular.

Given the abovementioned benefits of musicality, a link between musicality and vocal emotion perception seems plausible. We considered that individual musicality may be determined by a combination of genetic ("talent") and environmental factors ("training"), the contributions of which can be difficult to determine. Similarly, underlying mechanisms for the link between musicality and vocal emotion could be determined by both nature and nurture factors. On the nature side, some people might have an innate capacity to perceive fine-grained acoustic structures of both musical and vocal sounds, alongside with an inner drive to engage in musical activities. Accordingly, musical and vocal emotional capacities would be linked through genetic factors. This view is in line with Darwin's "protolanguage hypothesis", claiming that music and speech both evolved from the same origin, a musical protolanguage comprised of rudimental vocalizations (Darwin, 1871; Thompson et al., 2012), in which the expression of vocal emotions was a key aspect of communication (Fitch, 2013). Indeed, expression of emotion in music and vocal channels seem to be based on similar acoustic cues, supporting the idea that emotional communication in both channels are intertwined (Juslin \& Laukka, 2003). This gives rise to the possibility that capacities in both channels are driven by the same underlying genetic factors and that there are innate forces that create transfer between musical and vocal capacities.

On the nurture side, it is assumed that musical training causes the differences that are observed in musicians and non-musicians. From this perspective, the acoustic similarity between music and vocal emotions might be a reason for why extensive training in the musical domain can lead to an improvement in vocal emotion perception. A more elaborated nurture-based approach is offered by 
the OPERA hypothesis (Patel, 2011). Although it was originally developed to explain music-to-speech transfer effects, it can also be considered in the context of vocal emotion perception. The OPERA hypothesis states that musical-training benefits in other domains only occur when five conditions are met: (1) Overlap, (2) Precision, (3) Emotion, (4) Repetition and (5) Attention. Overlap refers to shared neural networks between music and vocal emotion processing. Indeed, neuroimaging data suggest common neural networks for the processing of emotional sounds, including vocal, musical and environmental sources (Escoffier et al., 2013; Frühholz et al., 2014; Frühholz et al., 2016; Grandjean, 2020; Schirmer et al., 2012). Core structures include the auditory cortex, the superior temporal cortex, frontal regions, the insula, the amygdala, the basal ganglia, and the cerebellum (Frühholz et al., 2016). Precision refers to the high auditory-motor demands that musical training places on these shared networks. The third condition, emotion, claims that the musical activity has to be perceived as rewarding. The subjective feeling of highly pleasurable experiences such as "chills", or "shivers-downthe-spine" are among the main reasons for why humans engage in musical activities, and those experiences are associated with brain activity changes in regions involved in reward, emotion, and arousal - including the amygdala, the ventral striatum, midbrain structures, orbitofrontal and ventromedial prefrontal areas (Blood \& Zatorre, 2001; Stewart et al., 2006). Finally, the necessities of repetition and attention stress the point that training-induced benefits depend critically on how frequently and focused musical activity is pursued over time. It may take years of active musical engagement to observe stable differences in musicians' brains (Kraus \& White-Schwoch, 2017), which can be regarded as truly reflecting training-induced changes (Elbert et al., 1995; Kraus \& Chandrasekaran, 2010; Pantev \& Herholz, 2011).

As it stands, the link of musicality with vocal emotion perception received relatively little attention and is poorly understood. This seems surprising, given that adequate emotion perception is crucial for wellbeing and perceived quality of life (Phillips et al., 2010; Schorr et al., 2009). However, to the best of our knowledge, there has been no attempt so far to integrate the existing evidence in a systematic manner. In this review, we aim at closing this gap, while including the full range of musical 
abilities: We survey findings from highly trained musicians, but also from people with exceptionally poor musical abilities. Finally, we include studies on cochlear implant $(\mathrm{Cl})$ users, who have significant difficulty recognizing vocal emotions due to degraded auditory input (Jiam et al., 2017), which might be improved with music-based interventions (Paquette et al., 2018).

\section{Systematic Literature Search / Inclusion Criteria}

We conducted parallel literature searches on Web of Science, PubMed, and PsychInfo on March 19, 2020, using the search terms "(voice OR prosody) AND (emotion* OR affect*) AND (music* OR auditory expert* OR amusi* OR auditory training)". We restricted publication language to English and considered empirical studies only. In total, the initial search returned 1723 articles (Web of Science N = 755, PubMed $N=405$ and PsychInfo $N=563$ ), to which we applied the following inclusion criteria: (1) vocal emotion perception was assessed as dependent variable, (2) musicality was assessed, manipulated, or used as a defining criterion for a group-based comparison, and (3) responses were measured on the behavioral or the brain level. We also screened the reference lists of the identified articles for relevant publications. This selection procedure resulted in a total of 33 articles, which we review below. For a summary, please refer to Table 1 . When screening the relevant literature, we noted that a substantial proportion (27/33, or $82 \%)$ of identified papers were published in 2011 or later, reflecting the current attention to this topic that contributed to motivating our present review.

[Insert Table 1 about here, please]

\section{Review of Identified Literature}

\section{Differences in Vocal Emotion Perception between Musicians and Non-musicians}

\section{Behavioral Data}

The presumably first study comparing vocal prosody perception in musicians and non-musicians comes from Nilsonne and Sundberg (1985). They presented pairs of fundamental frequency contours extracted from patients' voices while reading text either during depression or after recovery. 
Listeners were music and law students. Music students made significantly fewer errors than law students when judging which sample was recorded during depression. This study could be considered as first evidence for a musician effect on vocal emotion recognition, although depression obviously relates to more enduring affective states, relative to the more dynamic states typically communicated in emotional prosody as addressed in the studies below. These findings were replicated by Thompson et al. (2004). Musically trained participants performed better than untrained participants in categorizing emotional tone sequences extracted from vocal utterances. In a second Experiment however, which included additional sentences in familiar and unfamiliar languages, their effects were less straightforward: musicians' performance was better for sad, fearful, and neutral, but not for happy and angry prosody. As a caveat, musical training was associated with differences in cognitive abilities too, limiting conclusions from this study. This issue was addressed by Lima and Castro (2011), who compared highly trained musicians with non-musicians in two age groups (18-30 years and 40-60 years). Musicians outperformed their non-musical peers, similarly across emotions and age groups, even when effects were controlled for cognitive differences. Further, similar patterns of misattributions and acoustic cue utilization were observed in both groups, suggesting that group differences were of a quantitative rather than a qualitative nature.

Dmitrieva et al. (2006) tested vocal emotion perception by musicians and controls in children of three age groups (7-10, 11-13, and 14-17 years). Musicians outperformed their non-musical peers, but this effect was mainly driven by the youngest group. This finding could indicate either that the limited musical experience in very young children allowed innate aptitude to be more visible; or that musical experience might promote an earlier development of emotion sensitivity. Başkent et al. (2018) also studied adolescent musicians and non-musicians. Their work was motivated by Fuller et al. (2014), who conducted a similar design with adults. Both studies compared emotion recognition performance of unprocessed and degraded speech intended to reproduce the spectro-temporal degradation experienced by $\mathrm{Cl}$ users (Başkent et al., 2018). Whereas Fuller et al. (2014) found a small musician advantage in both conditions, Başkent et al. (2018) did not, potentially due to limited test- 
power with a much smaller sample compared to Fuller et al. (2014). Statistical comparison of both experiments revealed significant age effects, suggesting a maturation of the auditory system during emerging adulthood (Başkent et al., 2018).

Sensitivity to vocal prosody is of particular relevance in parent-infant interactions, where parents' adequate behavior crucially depends on their capacity to infer the infants' needs from the sound of their cries. Parsons et al. (2014) investigated sensitivity to distressed infants' cries in parents and non-parents. In parents, years of musical training was positively correlated with sensitivity to infant distress. Accordingly, musicality could foster a positive parent-child interaction due to enhanced sensitivity to the infants' emotional state. K. S. Young et al. (2012) used the same paradigm on musicians and non-musicians with and without depression. Musicians with depression performed comparable to non-depressed participants (with or without musical training) and significantly better than non-musicians with depression. Thus, musicality can potentially protect against compromised auditory sensitivity towards the infant during a depression period.

Several studies raised the question whether emotional sensitivity in musicians is restricted to the auditory domain. Twaite (2016) compared performance of musicians and non-musicians for prosodic, lexical, facial, and musical emotions and reported a musician advantage for the prosodic and the musical channel. No differences were observed for facial or lexical emotions, indicating that the musicians' advantage was limited to the auditory modality. However, Weijkamp and Sadakata (2017) reported somewhat conflicting results. They tested emotion recognition of musicians and nonmusicians in single-modality auditory and visual tasks, using voices and faces, as well as in an emotional Stroop task. No group differences were seen in the unimodal tasks, but musicians performed better in audiovisual tasks, which might point towards more efficient cross-modal integration. 
Up to now, most studies comparing musicians and non-musicians support the hypothesis that musicians have an advantage in vocal emotion perception. This advantage seems to be of a quantitative rather than a qualitative nature (Lima \& Castro, 2011; Twaite, 2016). However, the degree to which this advantage is moderated by factors such as innate musicality, the amount of musical training, age at training onset, or maturation of the auditory system all remain subjects for future research.

\section{Brain Data}

A small number of studies investigated differences between musicians and non-musicians with respect to the brain basis of vocal emotional processing. Strait et al. (2009) recorded brainstem potentials evoked by acoustically simple and complex portions of an infant's cry, and reported an intriguing interaction between musical expertise and stimulus complexity: Compared to controls, musicians showed reduced responses in the simple, but increased responses in the complex portion of the sound. The authors interpret these findings as indicating that (1) musical expertise results in fine neural tuning to acoustic features that are important to vocal communication, and (2) subcortical mechanisms contribute to vocal emotion perception. Further, different outcome measures in the brainstem signal suggested that the duration of musical training predicts temporal precision, whereas age at training onset was associated with fundamental frequency and timbre cues.

Using functional magnetic resonance imaging (fMRI), Park et al. (2015) compared brain activations of musicians and non-musicians to happy, sad, fearful, and neutral vocalizations. For sad stimuli only, musicians showed increased activation in the middle frontal gyrus, the anterior medial prefrontal cortex, the posterior cingulate cortex, and the retrosplenial cortex. The authors hypothesized that sadness might be of "higher affective saliency" for musicians.

Three electroencephalography (EEG) studies investigated how different cortical processing stages of vocal emotions differ as a function of musical expertise. Pinheiro et al. (2015) and Rigoulot et al. 
(2015) recorded event-related potentials (ERPs) and found that modulatory effects of musical expertise can be observed in early stages of cortical processing before $100 \mathrm{~ms}$ (P50, N100), as well as in later stages (P200). Nolden et al. (2017) re-analyzed the data of Rigoulot et al. (2015) with focus on induced oscillatory activity and found larger induced power for musicians in the theta (4-8 HZ) and the alpha-band (8-12 HZ).

Taken together, the existing work on brainstem potentials and EEG suggest that modulatory effects of musicality can be observed in very early processing steps of vocal emotions, which are associated with a basic analysis of auditory cues and allocation of emotional significance (Schirmer \& Kotz, 2006). Neuroimaging complements this by implicating brain regions associated with higher order functions. Overall, neuroscientific research on links between musicality and vocal emotion perception is still in its infancy, although it clearly has potential to shed light on the underlying mechanisms of musicians' enhanced ability to process vocal emotions.

\section{Effects of Musical Training Interventions on Vocal Emotion Perception}

Apart from comparing musicians and non-musicians, the effectiveness of musical interventions was at the focus of several studies, reviewed in this section. Note that designs with randomized assignments to intervention and control conditions are particularly valuable in the context of the nature-nurture debate, as they permit to deconfound training effects from self-selection effects when seeking musical education.

\section{Intervention-based Studies in Normal Hearing Individuals}

Thompson et al. (2004) randomly assigned six-year-old children to one year of training in keyboard, singing, drama, or no-lesson. Post-intervention, the drama and keyboard groups outperformed the no-lesson group in vocal emotion perception. Perhaps surprisingly, this effect was not found in the singing group. Thompson et al. (2004) speculated that singing may have trained vocal production of 
pitch contours that conflict with natural prosodic use of the voice. Nashkoff (2007) reported that simple pitch discrimination training alone can improve speech prosody decoding skills, but only for already highly trained musicians. Another attempt to show the effectiveness of musical intervention was made by Mualem and Lavidor (2015) who assigned participants either to music-based or visualart-based interventions, which focused explicitly on expression of emotions in the respective domain. After only four sessions, an improvement of vocal emotion recognition performance was observed in the music compared to the art group. However, when both groups were compared to a group of highly trained musicians, no performance differences were found. This could suggest that the effectiveness of the intervention partially reflected "training to the test", as the intervention explicitly focused on emotions. Finally, Bodner et al. (2012) reported that a music-based intervention improved recognition of happiness in patients with social anxiety disorder (SAD) who often display a persistent bias towards negative emotions. Although these findings need further verification, they suggest that perceptual biases in affective disorders may be attenuated by musical interventions. Together, the body of literature on interventions is still sparse for the normal hearing population, whereas musical trainings/interventions have been studied more intensely in the field of hearing rehabilitation for cochlear implant users.

\section{Intervention-based Studies in Cochlear Implant Users}

All studies reviewed in this section included vocal emotion perception as a part of larger test batteries to assess musical training effects on voice, speech and music perception in cochlear implant (Cl) users. Petersen et al. (2012) recruited $\mathrm{Cl}$ users within 14 days after implantation. Half of them received a six-month musical ear training. While distinct improvements were observed for musical perception, the pattern was less clear for vocal emotions: the intervention group showed an earlier onset of improvement, but the endpoints were comparable. However, the freshly implanted $\mathrm{Cl}$ users in this study were in speech therapy during the intervention, which could have interfered with the musical training. In contrast, Fuller et al. (2018) studied adult Cl users with a minimum of one year 
post implantation, who were randomly assigned to either (1) a pitch-timbre group that received receptive training, (2) a music-therapy group with face-to-face sessions including active music production, or (3) a control group with non-musical activities; over a period of six weeks. Crucially, vocal emotion recognition improved only in the music-therapy group, emphasizing the importance of active musical engagement and/or social interaction for training success. Similarly, Chari et al. (2020) also studied adult $\mathrm{Cl}$ users with at least one year of implant experience and assigned them to auditory-motor, auditory-only, or no training, over a period of three months. However, there was no effect on vocal emotion perception, even though the intervention period was about twice as long as in Fuller et al. (2018). Notably, both studies used very small sample sizes with less than 10 observations per group. While findings are intriguing and potentially important, they call for further exploration and replication with more powerful designs, particularly when effect sizes and statistical power are not (yet) routinely reported.

Only one study investigated the role of musical training in children with Cls, aged 6 and 15 years (Good et al., 2017). Improvements in vocal emotion perception were found after six months of piano lessons compared to a visual art training. The authors concluded that musical training might be an effective supplement for auditory rehabilitation in children. In addition to intervention-based approaches, Waaramaa et al. (2018) showed that self-reported musical interests - especially a preference for dancing - predicted vocal emotion perception capacity in $\mathrm{Cl}$ users. Taken together, findings emphasize an importance of active musical engagement, as compared to pure receptive training, in order to promote recovery of emotion perception after cochlear implantation.

\section{Impairments of Vocal Emotion Perception in Individuals with Congenital Amusia}

To gain a fuller understanding of the association between musicality and vocal emotion perception, it is also worthwhile to study individuals with exceptionally poor musical abilities, such as in congenital amusia. Individuals with congenital amusia display a perceptual disorder specific to the musical 
domain, in the presence of normal hearing and otherwise intact cognition (Ayotte et al., 2002;

Stewart et al., 2006). Congenital amusia is usually measured using the Montreal Battery of Evaluation of Amusia (MBEA, Peretz et al., 2003). Thompson et al. (2012) were the first to show poorer vocal prosody recognition in participants with amusia compared to controls and further observed a certain degree of awareness of their perceptual limitations in daily life. Lolli et al. (2015) suggested that a core problem could be poor pitch (F0) perception: although participants with suspected amusia performed similar to controls for emotion perception from unfiltered or high-pass filtered $(4800 \mathrm{~Hz})$ utterances, they performed poorer in low-pass filtered $(500 \mathrm{~Hz})$ utterances which presumably degraded timbre while preserving pitch contour information. Corroborating a selective deficit in pitch perception, Pralus et al. (2019) found that controls and participants with amusia exhibited comparable emotion recognition for whole sentences, but participants with amusia performed worse for vowels. Of relevance, perceived emotional intensity was comparable in both groups for all stimuli, which was interpreted as preserved implicit processing of emotional prosody in amusia.

Lima et al. (2016) took a crossmodal approach; in two experiments they tested adults with amusia and matched controls on their ability to identify emotions in different types of vocal stimuli and silent facial expressions. Participants with amusia were found to be impaired in the auditory and the visual domain, implying more universal emotion processing difficulties, potentially in line with an insightful analysis of patterns of category-specific but multimodal emotion recognition deficits that may also be characteristic of neuropsychological patients (A. W. Young, 2018). Zhang et al. (2018) and Cheung et al. (2020) were interested in relationships between amusia and emotional prosody processing in tonal languages. Zhang et al. (2018) tested participants from a Mandarin-speaking background with unfiltered and low-pass filtered speech $(500 \mathrm{~Hz})$. Unlike Lolli et al. (2015), participants with amusia were found to be worse for both filtered and unfiltered speech, disconfirming the hypothesis that tonal language acquisition might compensate for pitch-processing deficits in participants with amusia. For unfiltered speech, Cheung et al. (2020) reported similar findings in Cantonese speakers with amusia. Taken together, published findings on amusia paint a 
fairly consistent picture, suggesting that musical impairments transfer to vocal emotion perception, and that impairments for vocal emotions may originate from poor pitch perception.

\section{Correlation of Vocal Emotion Perception with Musical Interests or Psychoacoustic Abilities}

Complementing studies on extreme groups, other researchers measured normal interindividual variation of musicality in the general population to link it to variability in vocal emotion perception. The most compelling evidence against this link was provided by Trimmer and Cuddy (2008). Their correlational analysis for 100 participants revealed that musicality, as assessed via MBEA scores, was not associated with vocal emotion perception, a finding that they replicated with another 92 participants. Trimmer and Cuddy (2008) concluded that emotion perception in music and the voice is not linked via auditory sensitivity but rather via a supramodal emotional processor. This finding conflicts with many results discussed above and provoked large debates in the field. For instance, Lima and Castro (2011) argued that the participants in the study had only 6.5 years musical training on average, which might have been insufficient to observe a significant effect. However, Dibben et al. (2018) also failed to observe a relationship between musical interests and moment-to-moment reports of perceived emotion in longer (2-3 min) vocal excerpts. As a limitation, musical interests were assessed with a single dichotomous item in this study, which hardly captured fine grained interindividual variation of musicality.

Other studies reported positive correlations. Globerson et al. (2013) did not assess full-scale musical ability, but psychoacoustic measures of pitch-discrimination were found to predict vocal emotion perception performance. This highlights the importance of subtle pitch variations for emotional prosody perception, in line with the impairments in amusia discussed in the previous section. Finally, Waaramaa and Leisiö (2013) investigated the link between musical interests and emotional prosody perception in a large-scale cross-cultural study across five different countries (Estonia, Finland, Russia, Sweden, USA). Musical interests tended to have a positive effect on vocal emotion 
identification, but like in Dibben et al. (2018), this finding was based on very few self-reported items only.

In summary, correlational studies on the link between musicality and vocal emotion perception yielded conflicting results. This could be due to the substantial differences in assessing musicality, ranging from musical tests to short questionnaires. Indeed, the use of standardized and validated instruments for the assessment of musical interests is desirable, as this should promote better comparability across future studies, and contribute to resolving remaining controversies.

\section{Discussion}

While the transfer of musicality to speech perception abilities is well documented, the transfer to emotion perception attracted substantial scientific interest only recently. Overall, associations between musicality and vocal emotion perception ranged from strongly positive to absent, with the majority of studies supporting the idea that musicality is associated with better vocal emotion perception capacities. In the following, we will first discuss how these studies inform us about the contribution of nature and nurture factors for this link. Second, we will evaluate the effectiveness of active versus receptive musical training, and the moderating role of different acoustic cues signaling emotionality in both music and the voice. Finally, we will identify some relevant questions for further research.

\section{Nature and Nurture}

Musicality in people emerges from a combination of genetic and environmental factors. Likewise, the observed link between musicality and vocally expressed emotions could be either explained by a dispositional sensitivity to the musical and the vocal channel; or by a transfer from musical training effects into the vocal domain. Additionally, conditions of nature and nurture interact in individuals, making it difficult to estimate the degree of their respective contributions. Unfortunately, apart from 
few randomized treatment studies that potentially isolated training effects, all reviewed papers established correlational designs or studied pre-existing groups and thus cannot provide direct evidence for the relative contributions of nature or nurture conditions. Nevertheless, it is worthwhile to consider implications of certain findings for this debate.

Without exception, all the studies on people with amusia suggested that vocal emotion perception deficits can be associated with a congenital music perception impairment. In that sense, the link between musicality and vocal emotion perception seems to occur in the absence of training effects and might therefore be mediated by genetic factors. These may have evolved in parallel with acoustic similarities between vocal and musical emotions (Juslin \& Laukka, 2003) and may be expressed in overlapping neural circuits involved in recognizing basic emotions from voices and music (see also Frühholz et al., 2016). As a qualification, Bigand and Poulin-Charronnat (2006) showed that a remarkable degree of auditory sophistication can be acquired through exposure to music only, without explicit training. Accordingly, it remains possible that these implicit musical learning processes could be limited in people with amusia if they avoid exposure to music because they enjoy it less. Thus, while the limited vocal emotion capacities observed in amusia could hint towards a genetic predisposition, they could also result in part from selective exposure.

At the same time, a consistent set of findings from highly trained musicians suggests that explicit musical training does play a central role for the development of auditory and vocal perceptual skills. This points towards an influence of environmental factors, but there is always a possible confound with natural inclination, as people with better auditory skills may be more likely to start and pursue musical training (Pantev \& Herholz, 2011). Accordingly, Dmitrieva et al. (2006) observed superior vocal emotion capacities in a very young group of musicians, who presumably had very little musical training yet but might have been selected for musical education based on their auditory sensitivity. Note that many authors who found the musician effect on vocal emotion perception argued that it is very unlikely that it is entirely based on predispositional differences (Lima \& Castro, 2011; Strait et al., 2009; Thompson et al., 2004): On the one hand, the effect was still present when participants 
were matched in socio-educational variables, general intelligence, cognitive control, and personality traits (Lima \& Castro, 2011). On the other hand, some studies found a correlation between emotion perception capacities and years of musical education, suggesting a clear impact of training duration (Parsons et al., 2014; Twaite, 2016; K. S. Young et al., 2012). However, this could also reflect a geneenvironment interaction since people who have a dispositional aptitude might stick longer with the training. Further, vocal emotion perception was found to be related to age at training onset (Strait et al., 2009). Although it is often difficult to disentangle age at onset with years of musical training, this could suggest a sensitive period for the acquisition of some music-training induced skills. Accordingly, many studies required musicians to have started training before the age of seven (refer to Table 1).

Finally, a few intervention studies with randomized assignment to treatment and control groups aimed at isolating learning effects of musical training and succeeded to improve vocal emotion perception in the healthy population. Note that these interventions were qualitatively different to more "natural" settings of musical education, where the focus lies on mastery of an instrument or the singing voice. They were shorter and often particularly focused on emotion expression in music (Bodner et al., 2012; Mualem \& Lavidor, 2015), apart from Thompson et al. (2004), who randomly assigned children to one year of keyboard and singing lessons, but found mixed results. Likewise, studies on cochlea implant users showed that musical training can improve vocal emotion perception in this particular group; but again, those interventions had an entirely different purpose than in normal hearing participants: instead of fine-tuning a healthy auditory system, $\mathrm{Cl}$ users have to learn how to restore perception from a severely degraded input. Music-based interventions may be particularly effective in groups with poor auditory resolution to improve sensitivity to auditory cues in the vocal domain (Fuller et al., 2018; Good et al., 2017). Overall, while it may be difficult to generalize the results of these intervention studies to settings of instrumental or vocal music lessons, they show that vocal emotion perception is amenable to musical training in some circumstances. Accordingly, it seems worthwhile to incorporate emotionally oriented teaching units in music lessons or treatment programs. 


\section{Active Engagement in Musical Activities vs Receptive Training}

Several studies suggest that active engagement in a musical task is a crucial factor. They compared purely receptive training to auditory-motor training and reported stable benefits in the vocal emotion domain for auditory-motor training (Chari et al., 2020; Fuller et al., 2018). There is high consensus in the neuroscientific literature, that active engagement in music and the synchronized tuning of auditory, visual, somatosensory, and motor processes is a driving force to adaptive neuroplasticity (Kraus \& Chandrasekaran, 2010; Kraus \& White-Schwoch, 2017; Palomar-García et al., 2017). Specifically, it has been shown that sensorimotor musical training leads to more robust changes in the auditory cortex compared to pure receptive training (Lappe et al., 2008). This surely does not imply that purely receptive music training is ineffective (Bigand \& Poulin-Charronnat, 2006) - but motor engagement may add a boost to the auditory fine-tuning process during training. This could be of particular relevance for cochlear implant users (Lehmann \& Paquette, 2015), who during rehabilitation face the challenge of massive post-implantation adaptation to the new auditory input. Here, auditory-motor interventions could be particularly efficient to foster neuroplasticity in auditory areas and aid hearing rehabilitation.

\section{The Role of Different Acoustic Cues and Supramodal Processes}

Previous literature suggests that musicians show superior processing of pitch, temporal and timbral cues (Elmer et al., 2018). The present review reveals that superior pitch processing capacities in people with high levels of musicality are particularly tightly associated with vocal emotion perception. On the one hand, pitch discrimination performance was correlated with emotion perception performance in musicians; on the other hand, there was strong agreement that impaired pitch processing was a key deficit in people with amusia accounting for impairments in the domain of vocal emotions. However, this conclusion has its limitations, since amusia was often defined only based on low scores in the pitch subsets of the MBEA (refer to Table 1). According to Juslin and 
Laukka (2003), pitch and timbral cues are highly relevant for vocal emotion perception, but timing parameters like speech rate were found to be equally important. The potential role of timing was largely neglected in all reviewed studies, despite its central role in music, therein often referred to as tempo and rhythm. Lagrois and Peretz (2019) showed that pitch and rhythm deficits are often linked in people with amusia, but they sometimes can appear as distinct disorders. In parallel, there is current evidence for different brain mechanisms to process harmonic versus rhythmic structures in music (Sun et al., 2020). In the future, it would be very informative to investigate vocal emotion perception in people with specific impairments in the temporal domain of music.

Alongside the notion that enhanced sensitivity to acoustic cues may lead to better emotion perception in people with higher level of musicality, it was also suggested that there might be a domain-general supramodal process that mediates the link between musicality and emotional perception across domains (Schellenberg \& Mankarious, 2012; Trimmer \& Cuddy, 2008). Lima and Castro (2011) suggested that musical training might increase the level of "emotional granularity", meaning a more fine-grained conceptualization and differentiation of emotions, that in turn could aid emotional perception in other domains. However, although the involvement of supramodal processes seems plausible, the reviewed brain data suggest that modulatory effects of musicality can be observed in very early processing steps of vocal emotions, which are associated with a basic analysis of auditory cues and detection of emotional saliency (Schirmer \& Kotz, 2006). Hence, the link between musicality and vocal emotion perception seems to be based, at least partially, on a more sophisticated analysis of auditory cues.

\section{Identification of Relevant Topics for Future Research}

The findings surveyed in this paper highlight many relevant aspects that can guide future research regarding relationships between musicality and vocal emotion perception. We hope this article will inform systematic research programs with better powered designs and standardized research 
materials, and ultimately promote a refined understanding of the putative common mechanisms underlying musicality and vocal emotion perception. Unfortunately, neuroscientific research in this field is still sparse and unsystematic, and the heterogeneity of the above studies illustrates the need for more systematic research on candidate subcortical and cortical mechanisms to mediate the link between musicality and vocal emotion perception. Important questions for neuroscientific research include how perceptual neuroplasticity is induced in musicians, how this relates to motor plasticity in musicians' brains (Elbert et al., 1995), what are the relative roles of training or ongoing maintenance (Merrett et al., 2013), and how each of these aspects relates to vocal emotion perception. Moreover, a particularly relevant comparison in the context of vocal emotion perception is the one between singers and instrumentalists. Most of the reviewed studies only included instrumentalists or did not report on that matter. Only Thompson et al. (2004) compared participants with piano and singing lessons, and their results suggested that the singing lessons might even hinder vocal emotion perception, perhaps because the vocal patterns that are trained during singing lessons may conflict with natural vocal emotion expression. Another neglected but related field is the link between musicality and emotion production. It may be reasonable to assume that people who are highly trained in an emotionally expressive art have an advantage in vocal expression of emotion.

\section{Conclusion and Outlook}

In this review, we systematically identified and discussed the current state of research regarding a link between musicality and vocal emotion perception. Overall, the available evidence suggests that musicality is indeed associated with better vocal emotion perception performance. Since adequate perception of vocal emotions forms a fundamental prerequisite for everyday social interaction, these results also may add weight to the presumed importance of music and musical education for personal development and quality of life. Musical training can provide a promising supplemental intervention for people who struggle with vocal emotion perception, and while supporting evidence can now be considered strong in the case of cochlear implant users, future applied research seems 
promising in the context of other target groups as well (e.g., individuals with autism, or with highlevel auditory impairments). Although data often do not allow for causal inferences, their combined consideration can provide useful information for the question of how different factors of nature and nurture contribute to related skills of emotion perception in the domains of voice and music.

\section{Acknowledgements}

C.N. research has been supported by the German National Academic Foundation ("Studienstiftung des Deutschen Volkes"). We thank Annett Schirmer for her helpful and critical comments. We are grateful to Susan Müller and Frank Nussbaum for their support on the manuscript.

\section{Conflicts of Interests}

The authors declare no conflicts of interests.

\section{References}

Aubé, W., Angulo-Perkins, A., Peretz, I., Concha, L., \& Armony, J. L. (2015). Fear across the senses: brain responses to music, vocalizations and facial expressions. Social Cognitive and Affective Neuroscience, 10(3), 399-407.

Ayotte, J., Peretz, I., \& Hyde, K. (2002). Congenital amusia: A group study of adults afflicted with a music-specific disorder. Brain : A Journal of Neurology, 125(Pt 2), 238-251.

https://doi.org/10.1093/brain/awf028

Başkent, D., Fuller, C. D., Galvin, J. J., Schepel, L., Gaudrain, E., \& Free, R. H. (2018). Musician effect on perception of spectro-temporally degraded speech, vocal emotion, and music in young adolescents. The Journal of the Acoustical Society of America, 143(5), EL311. https://doi.org/10.1121/1.5034489 
Bigand, E., \& Poulin-Charronnat, B. (2006). Are we "experienced listeners"? A review of the musical capacities that do not depend on formal musical training. Cognition, 100(1), 100-130.

Blood, A. J., \& Zatorre, R. J. (2001). Intensely pleasurable responses to music correlate with activity in brain regions implicated in reward and emotion. Proceedings of the National Academy of Sciences, 98(20), 11818-11823.

Bodner, E., Aharoni, R., \& lancu, I. (2012). The Effect of Training with Music on Happiness Recognition in Social Anxiety Disorder. Journal of Psychopathology and Behavioral Assessment, 34(4), 458466. https://doi.org/10.1007/s10862-012-9304-7

Chari, D. A., Barrett, K. C., Patel, A. D [Aniruddh D.], Colgrove, T. R., Jiradejvong, P., Jacobs, L. Y., \& Limb, C. J [Charles J.] (2020). Impact of Auditory-Motor Musical Training on Melodic Pattern Recognition in Cochlear Implant Users. Otology \& Neurotology : Official Publication of the American Otological Society, American Neurotology Society [and] European Academy of Otology and Neurotology, 41(4), e422-e431. https://doi.org/10.1097/MA0.0000000000002525

Chartrand, J.-P., Peretz, I., \& Belin, P. (2008). Auditory recognition expertise and domain specificity. Brain Research, 1220, 191-198.

Cheung, Y. L., Zhang, C., \& Zhang, Y [Yubin] (2020). Emotion processing in congenital amusia: The deficits do not generalize to written emotion words. Clinical Linguistics \& Phonetics, 1-16. https://doi.org/10.1080/02699206.2020.1719209

Darwin, C. (1871). Sexual selection and the descent of man. London: Murray, 289, 290.

Dibben, N., Coutinho, E., Vilar, J. A., \& Estévez-Pérez, G. (2018). Do Individual Differences Influence Moment-by-Moment Reports of Emotion Perceived in Music and Speech Prosody? Frontiers in Behavioral Neuroscience, 12, 184. https://doi.org/10.3389/fnbeh.2018.00184

Dmitrieva, E. S., Gel'man, V. Y., Zaitseva, K. A., \& Am Orlov (2006). Ontogenetic features of the psychophysiological mechanisms of perception of the emotional component of speech in musically gifted children. Neuroscience and Behavioral Physiology, 36(1), 53. 
Elbert, T., Pantev, C., Wienbruch, C., Rockstroh, B., \& Taub, E. (1995). Increased cortical representation of the fingers of the left hand in string players. Science, $270(5234), 305-307$.

Elmer, S., Dittinger, E., \& Besson, M. (2018). One Step Beyond: Musical Expertise and Word Learning. The Oxford Handbook of Voice Perception, 209-235.

Escoffier, N., Zhong, J., Schirmer, A., \& Qiu, A. (2013). Emotional expressions in voice and music: same code, same effect? Human Brain Mapping, 34(8), 1796-1810.

Fitch, W. T. (2013). Musical protolanguage: Darwin's theory of language evolution revisited. Birdsong, Speech, and Language: Exploring the Evolution of Mind and Brain, 489, 503.

Frühholz, S., Trost, W., \& Grandjean, D. (2014). The role of the medial temporal limbic system in processing emotions in voice and music. Progress in Neurobiology, 123, 1-17. https://doi.org/10.1016/j.pneurobio.2014.09.003

Frühholz, S., Trost, W., \& Kotz, S. A. (2016). The sound of emotions-Towards a unifying neural network perspective of affective sound processing. Neuroscience \& Biobehavioral Reviews, 68, 96-110.

Fuller, C. D., Galvin, J. J., Maat, B., Başkent, D., \& Free, R. H. (2018). Comparison of Two Music Training Approaches on Music and Speech Perception in Cochlear Implant Users. Trends in Hearing, 22, 2331216518765379. https://doi.org/10.1177/2331216518765379

Fuller, C. D., Galvin, J. J., Maat, B., Free, R. H., \& Başkent, D. (2014). The musician effect: Does it persist under degraded pitch conditions of cochlear implant simulations? Frontiers in Neuroscience, 8, 179. https://doi.org/10.3389/fnins.2014.00179

Globerson, E., Amir, N., Golan, O., Kishon-Rabin, L., \& Lavidor, M. (2013). Psychoacoustic abilities as predictors of vocal emotion recognition. Attention, Perception \& Psychophysics, 75(8), 1799-1810. https://doi.org/10.3758/s13414-013-0518-x

Good, A., Gordon, K. A., Papsin, B. C., Nespoli, G., Hopyan, T., Peretz, I., \& Russo, F. A. (2017). Benefits of Music Training for Perception of Emotional Speech Prosody in Deaf Children With Cochlear Implants. Ear and Hearing, 38(4), 455-464. https://doi.org/10.1097/AUD.0000000000000402 
Grandjean, D. (2020). Brain networks of emotional prosody processing. Emotion Review, 1754073919898522.

Jiam, N. T., Caldwell, M., Deroche, M. L., Chatterjee, M., \& Limb, C. J [C. J.] (2017). Voice emotion perception and production in cochlear implant users. Hear Res, 352, 30-39. https://doi.org/10.1016/j.heares.2017.01.006

Juslin, P. N., \& Laukka, P. (2003). Communication of emotions in vocal expression and music performance: different channels, same code? Psychol Bull, 129(5), 770-814. https://doi.org/10.1037/0033-2909.129.5.770

Kraus, N., \& Chandrasekaran, B. (2010). Music training for the development of auditory skills. Nature Reviews Neuroscience, 11(8), 599-605.

Kraus, N., \& White-Schwoch, T. (2017). Neurobiology of Everyday Communication: What Have We Learned From Music? The Neuroscientist : A Review Journal Bringing Neurobiology, Neurology and Psychiatry, 23(3), 287-298. https://doi.org/10.1177/1073858416653593

Lagrois, M.-É., \& Peretz, I. (2019). The co-occurrence of pitch and rhythm disorders in congenital amusia. Cortex, 113, 229-238.

Lappe, C., Herholz, S. C., Trainor, L. J., \& Pantev, C. (2008). Cortical plasticity induced by short-term unimodal and multimodal musical training. Journal of Neuroscience, 28(39), 9632-9639.

Lehmann, A [Alexandre], \& Paquette, S [Sébastien] (2015). Cross-domain processing of musical and vocal emotions in cochlear implant users. Frontiers in Neuroscience, 9, 343.

https://doi.org/10.3389/fnins.2015.00343

Lima, C. F., Brancatisano, O., Fancourt, A., Müllensiefen, D., Scott, S. K., Warren, J. D., \& Stewart, L [Lauren] (2016). Impaired socio-emotional processing in a developmental music disorder. Scientific Reports, 6, 34911. https://doi.org/10.1038/srep34911

Lima, C. F., \& Castro, S. L. (2011). Speaking to the trained ear: musical expertise enhances the recognition of emotions in speech prosody. Emotion, 11(5), 1021-1031. https://doi.org/10.1037/a0024521 
Lolli, S. L., Lewenstein, A. D., Basurto, J., Winnik, S., \& Loui, P. (2015). Sound frequency affects speech emotion perception: Results from congenital amusia. Frontiers in Psychology, 6, 1340. https://doi.org/10.3389/fpsyg.2015.01340

Merrett, D. L., Peretz, I., \& Wilson, S. J. (2013). Moderating variables of music training-induced neuroplasticity: a review and discussion. Frontiers in Psychology, 4, 606.

Mill, A., Allik, J., Realo, A., \& Valk, R. (2009). Age-related differences in emotion recognition ability: A cross-sectional study. Emotion, 9(5), 619.

Mualem, O., \& Lavidor, M. (2015). Music education intervention improves vocal emotion recognition. International Journal of Music Education, 33(4), 413-425.

https://doi.org/10.1177/0255761415584292

Nashkoff, K. (2007). The relationship between pitch discrimination skills and speech prosody decoding skills [, Walden University]. EndNote Tagged Import Format.

Nilsonne, Å., \& Sundberg, J. (1985). Differences in Ability of Musicians and Nonmusicians to Judge Emotional State from the Fundamental Frequency of Voice Samples. Music Perception: An Interdisciplinary Journal, 2(4), 507-516. https://doi.org/10.2307/40285316

Nolden, S., Rigoulot, S., Jolicoeur, P., \& Armony, J. L. (2017). Effects of musical expertise on oscillatory brain activity in response to emotional sounds. Neuropsychologia, 103, 96-105. https://doi.org/10.1016/j.neuropsychologia.2017.07.014

Palomar-García, M.-Á., Zatorre, R. J., Ventura-Campos, N., Bueichekú, E., \& Ávila, C. (2017). Modulation of Functional Connectivity in Auditory-Motor Networks in Musicians Compared with Nonmusicians. Cerebral Cortex (New York, N.Y. : 1991), 27(5), 2768-2778. https://doi.org/10.1093/cercor/bhw120

Pantev, C., \& Herholz, S. C. (2011). Plasticity of the human auditory cortex related to musical training. Neuroscience and Biobehavioral Reviews, 35(10), 2140-2154. https://doi.org/10.1016/j.neubiorev.2011.06.010 
Paquette, S [S.], Ahmed, G. D., Goffi-Gomez, M. V., Hoshino, A. C.H., Peretz, I., \& Lehmann, A [A.] (2018). Musical and vocal emotion perception for cochlear implants users. Hearing Research, 370, $272-282$

Park, M., Gutyrchik, E., Welker, L., Carl, P., Pöppel, E., Zaytseva, Y., Meindl, T., Blautzik, J., Reiser, M., \& Bao, Y. (2015). Sadness is unique: neural processing of emotions in speech prosody in musicians and non-musicians. Frontiers in Human Neuroscience, 8, 1049.

Parsons, C. E., Young, K. S., Jegindø, E.-M. E., Vuust, P., Stein, A., \& Kringelbach, M. L. (2014). Music training and empathy positively impact adults' sensitivity to infant distress. Frontiers in Psychology, 5, 1440. https://doi.org/10.3389/fpsyg.2014.01440

Patel, A. D [A. D.] (2011). Why would Musical Training Benefit the Neural Encoding of Speech? The OPERA Hypothesis. Front Psychol, 2, 142. https://doi.org/10.3389/fpsyg.2011.00142

Peretz, I., Champod, A. S., \& Hyde, K. (2003). Varieties of musical disorders: the Montreal Battery of Evaluation of Amusia. Annals of the New York Academy of Sciences, 999(1), 58-75.

Petersen, B., Mortensen, M. V., Hansen, M., \& Vuust, P. (2012). Singing in the key of life: A study on effects of musical ear training after cochlear implantation. Psychomusicology: Music, Mind, and Brain, 22(2), 134-151. https://doi.org/10.1037/a0031140

Phillips, L. H., Scott, C., Henry, J. D., Mowat, D., \& Bell, J. S. (2010). Emotion perception in Alzheimer's disease and mood disorder in old age. Psychology and Aging, 25(1), 38.

Pinheiro, A. P., Vasconcelos, M., Dias, M., Arrais, N., \& Gonçalves, Ó. F. (2015). The music of language: an ERP investigation of the effects of musical training on emotional prosody processing. Brain Lang, 140, 24-34. https://doi.org/10.1016/j.bandl.2014.10.009

Pralus, A., Fornoni, L., Bouet, R., Gomot, M., Bhatara, A., Tillmann, B., \& Caclin, A. (2019). Emotional prosody in congenital amusia: Impaired and spared processes. Neuropsychologia, 134, 107234. https://doi.org/10.1016/j.neuropsychologia.2019.107234 
Rigoulot, S., Pell, M. D., \& Armony, J. L. (2015). Time course of the influence of musical expertise on the processing of vocal and musical sounds. Neuroscience, 290, 175-184. https://doi.org/10.1016/j.neuroscience.2015.01.033

Sauter, D. A. (2017). The nonverbal communication of positive emotions: An emotion family approach. Emotion Review, 9(3), 222-234.

Schäfer, T., Sedlmeier, P., Städtler, C., \& Huron, D. (2013). The psychological functions of music listening. Frontiers in Psychology, 4, 511.

Schellenberg, E. G [E. Glenn] (2001). Music and nonmusical abilities. Annals of the New York Academy of Sciences, 930(1), 355-371.

Schellenberg, E. G [E. Glenn] (2016). Music training and nonmusical abilities. The Oxford Handbook of Music Psychology, 2, 415-429.

Schellenberg, E. G [E. Glenn], \& Mankarious, M. (2012). Music training and emotion comprehension in childhood. Emotion (Washington, D.C.), 12(5), 887-891. https://doi.org/10.1037/a0027971

Scherer, K. R. (1986). Vocal affect expression: a review and a model for future research. Psychol Bull, $99(2), 143-165$.

Scherer, K. R. (1995). Expression of emotion in voice and music. Journal of Voice, 9(3), 235-248.

Scherer, K. R., Banse, R., \& Wallbott, H. G. (2016). Emotion Inferences from Vocal Expression Correlate Across Languages and Cultures. Journal of Cross-Cultural Psychology, 32(1), 76-92. https://doi.org/10.1177/0022022101032001009

Schirmer, A., Fox, P. M., \& Grandjean, D. (2012). On the spatial organization of sound processing in the human temporal lobe: a meta-analysis. Neuroimage, 63(1), 137-147.

Schirmer, A., \& Kotz, S. A. (2006). Beyond the right hemisphere: brain mechanisms mediating vocal emotional processing. Trends Cogn Sci, 10(1), 24-30. https://doi.org/10.1016/j.tics.2005.11.009

Schirmer, A., Striano, T., \& Friederici, A. D. (2005). Sex differences in the preattentive processing of vocal emotional expressions. Neuroreport, 16(6), 635-639. 
Schorr, E. A., Roth, F. P., \& Fox, N. A. (2009). Quality of life for children with cochlear implants: Perceived benefits and problems and the perception of single words and emotional sounds. Journal of Speech, Language, and Hearing Research.

Stewart, L [Lauren], Kriegstein, K. von, Warren, J. D., \& Griffiths, T. D. (2006). Music and the brain: disorders of musical listening. Brain : A Journal of Neurology, 129(10), 2533-2553.

Strait, D. L., Kraus, N., Skoe, E., \& Ashley, R. (2009). Musical experience and neural efficiency: Effects of training on subcortical processing of vocal expressions of emotion. The European Journal of Neuroscience, 29(3), 661-668. https://doi.org/10.1111/j.1460-9568.2009.06617.x

Sun, L., Thompson, W. F [William Forde], Liu, F., Zhou, L., \& Jiang, C. (2020). The human brain processes hierarchical structures of meter and harmony differently: Evidence from musicians and nonmusicians. PSYCHOPHYSIOLOGY, e13598.

Thompson, W. F [W. F.], Marin, M. M., \& Stewart, L [L.] (2012). Reduced sensitivity to emotional prosody in congenital amusia rekindles the musical protolanguage hypothesis. Proc Natl Acad Sci U S a, 109(46), 19027-19032. https://doi.org/10.1073/pnas.1210344109

Thompson, W. F [W. F.], Schellenberg, E. G [E. G.], \& Husain, G. (2004). Decoding speech prosody: do music lessons help? Emotion, 4(1), 46-64. https://doi.org/10.1037/1528-3542.4.1.46

Trimmer, C. G., \& Cuddy, L. L. (2008). Emotional intelligence, not music training, predicts recognition of emotional speech prosody. Emotion (Washington, D.C.), 8(6), 838-849. https://doi.org/10.1037/a0014080

Twaite, J. (2016). Examining Relationships Between Basic Emotion Perception and Musical Training in the Prosodic, Facial, and Lexical Channels of Communication and in Music.

Waaramaa, T., Kukkonen, T., Mykkänen, S., \& Geneid, A. (2018). Vocal Emotion Identification by Children Using Cochlear Implants, Relations to Voice Quality, and Musical Interests. Journal of Speech, Language, and Hearing Research : JSLHR, 61(4), 973-985.

https://doi.org/10.1044/2017_JSLHR-H-17-0054 
Waaramaa, T., \& Leisiö, T. (2013). Perception of emotionally loaded vocal expressions and its connection to responses to music. A cross-cultural investigation: Estonia, Finland, Sweden, Russia, and the USA. Frontiers in Psychology, 4, 344. https://doi.org/10.3389/fpsyg.2013.00344

Weijkamp, J., \& Sadakata, M. (2017). Attention to affective audio-visual information: Comparison between musicians and non-musicians. Psychology of Music, 45(2), 204-215. https://doi.org/10.1177/0305735616654216

Young, A. W. (2018). Faces, people and the brain: the 45th Sir Frederic Bartlett Lecture. The Quarterly Journal of Experimental Psychology, 71(3), 569-594.

Young, A. W., Frühholz, S., \& Schweinberger, S. R. (2020). Face and voice perception: Understanding commonalities and differences. Trends in Cognitive Sciences.

Young, K. S., Parsons, C. E., Stein, A., \& Kringelbach, M. L. (2012). Interpreting infant vocal distress: The ameliorative effect of musical training in depression. Emotion (Washington, D.C.), 12(6), 1200-1205. https://doi.org/10.1037/a0028705

Zhang, Y [Yixin], Geng, T., \& Zhang, J. (2018, September). Emotional Prosody Perception in Mandarinspeaking Congenital Amusics. In Interspeech 2018 (pp. 2196-2200). ISCA.

https://doi.org/10.21437/Interspeech.2018-91 
Table 1: Summary of empirical articles on musicality and vocal emotion perception that met the selection criteria

\begin{tabular}{|c|c|c|c|c|c|}
\hline Reference & Sample (Mean Age) & Inclusion Criteria & Stimuli and Task & Emotions & Results \\
\hline Musical Expertise & & Musicians & & & \\
\hline \multirow[t]{4}{*}{ Başkent et al., 2018} & 10 musicians (12 y), 11 & training onset before age 7 & original and degraded speech, & joy, anger, sadness, & musician effect only in melodic contour \\
\hline & non-musicians (12 y) & yrs, $>5$ yrs of musical & melodic contour identification, & and relief & identification in degraded speech condition \\
\hline & & training, regular training & vocal emotion identification, and & & \\
\hline & & within the last 3 yrs & speech understanding in noise & & \\
\hline Dmitrieva et al., & 48 musicians, 46 & recruited from the Russian & vocal emotion identification, & joy, anger, and & Musicians were better and faster in emotion \\
\hline \multirow[t]{3}{*}{2006} & controls ( 3 age groups: & National Orchestra, St. & presentation to one ear with & neutral & recognition \\
\hline & $7-10 y, 11-13 y$, and & Petersburg & white noise on the other & & \\
\hline & $14-17$ y) & & & & \\
\hline \multirow[t]{4}{*}{ Fuller et al., 2014} & 25 musicians ( 23 y), 25 & training onset before age 7 & original and degraded (8-channel & joy, anger,sadness, & musicians better in emotion recognition in both \\
\hline & non-musicians (22 y) & yrs, $>10$ yrs of musical & simulated ) stimuli, tests on & and relief & original and degraded condition \\
\hline & & training, regular training & speech, vocal emotion, and & & \\
\hline & & within the last 3 yrs & melodic contour identification & & \\
\hline \multirow[t]{4}{*}{ Lima \& Castro, 2011} & 40 musicians, 40 non- & instrumentalists, $>8$ years & vocal emotion identification and & happines, anger, & musicians were better in emotion recognition, \\
\hline & musicians ( 2 age & of musical training, & intensity rating & fear, sadness, & controlled for cognitive differences \\
\hline & groups: $18-30$ y and $40-$ & started in childhood, & & surprise, disgust, & \\
\hline & 60 y) & current regular practise & & and neutral & \\
\hline
\end{tabular}

Note: $V E R=$ vocal emotion recognition, MBEA = Montreal Battery of Evaluation of Amusia (Peretz et al., 2003) 
Table 1: Summary of empirical articles on musicality and vocal emotion perception that met the selection criteria continued

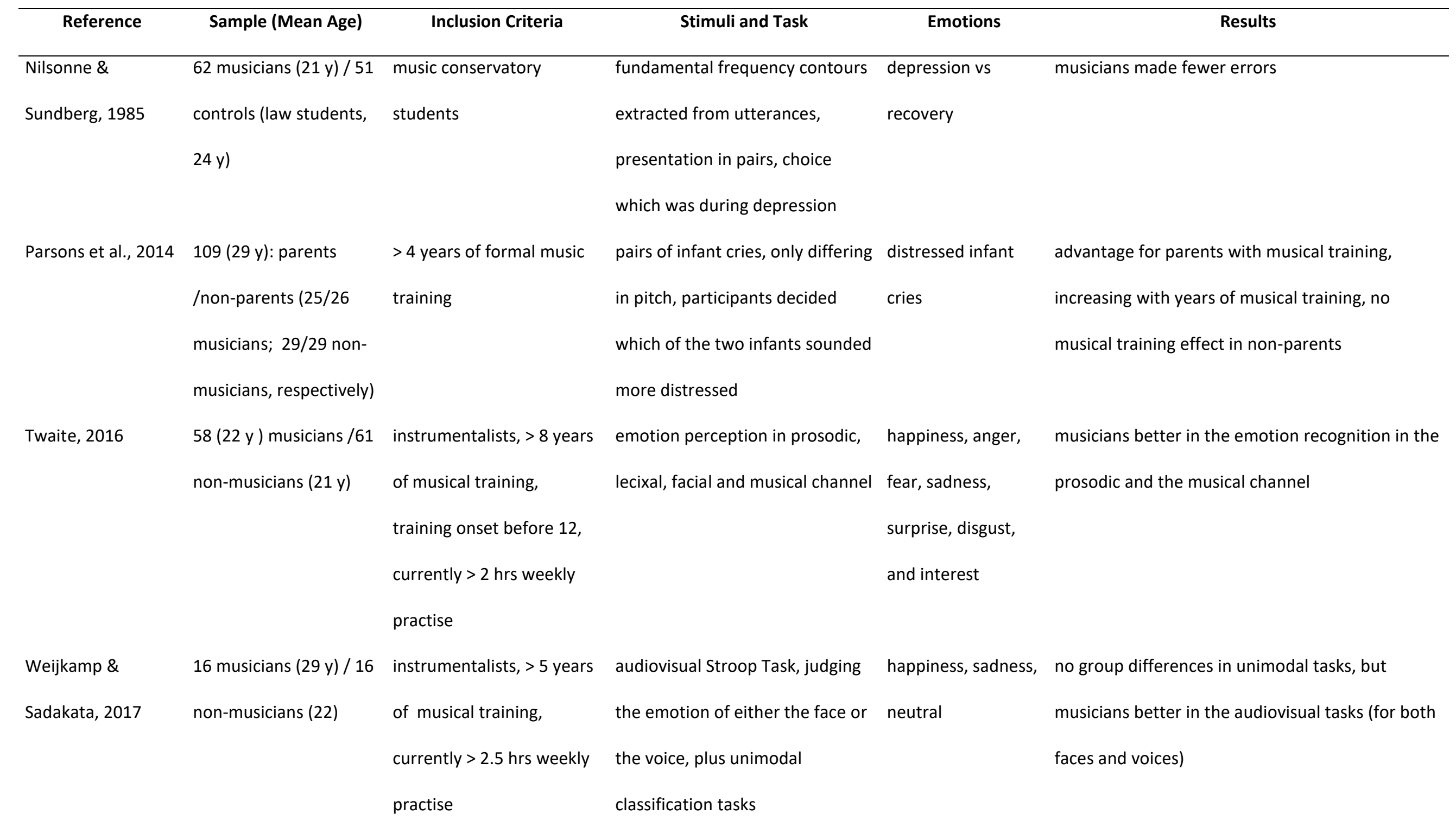


Table 1: Summary of empirical articles on musicality and vocal emotion perception that met the selection criteria continued

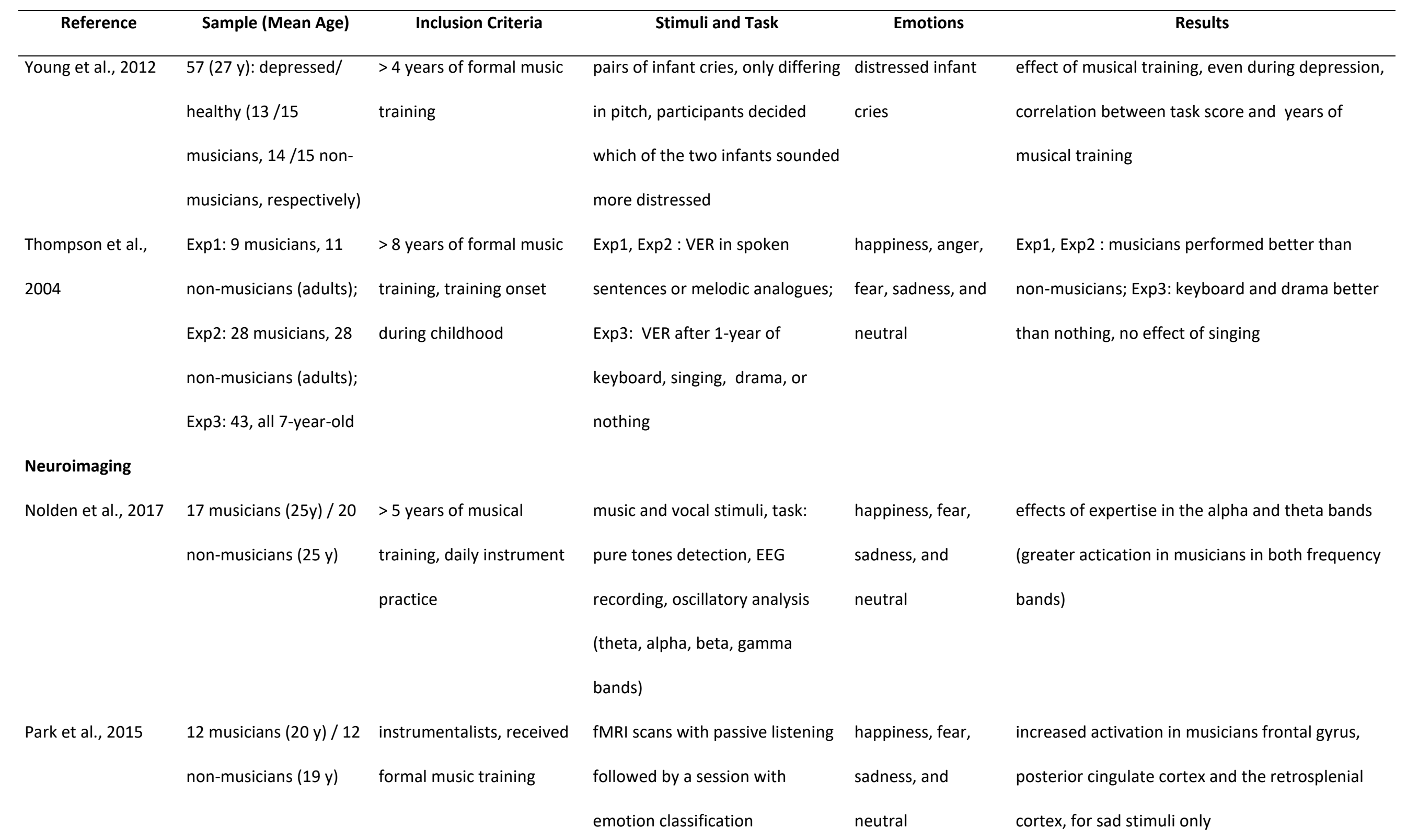


Table 1: Summary of empirical articles on musicality and vocal emotion perception that met the selection criteria continued

\begin{tabular}{llll}
\hline Reference & Sample (Mean Age) & Inclusion Criteria & Stimuli and Task
\end{tabular}

(mean years of training $=$

$13.8, \mathrm{SD}=2.6)$

Pinheiro et al., 201514 musicians ( 23 y) / $14>8$ years musical training, emotion classifiction with non-musicians (23 y) daily instrument practice

Rigoulot et al., 201515 musicians ( 24 y) / $18>5$ years of musical non-musicians (25 y) training, daily instrument practice

Strait et al., 2009

30 adults $(25 y)$ musicians (by onset 'musicians by onset age': training onset before age age: $\mathrm{n}=11$; by years: $\mathrm{n} \quad 7$, 'musicians by years': > $=15) /$ non-musicians $(\mathrm{n} \quad 10$ years musical training $=19 / 15$, respectively)

\section{Musical training /intervention}

Bodner et al., 201280 (29 y): 39 with social anxiety disorder, 41 healthy controls intelligible semantic content (SCC) or unintelligible semantic content (PPC), EEG recording

happiness, anger

P50 more positive in controls than musician in SCC

and neutral only, musicians better in recognition of anger in

PCC only

music and vocal stimuli, task:

pure tones detection, EEG

happiness, fear sadness, and

neutral

infant cries

exposure to infants unhappy

cries, recording of auditory

brainstem responses, during a

complex and more periodic part

of the stimulus

Intervention: training of

happiness, anger

music intervention improved recognition of vocal

happiness recognition in music,

fear, sadness, and

happiness in participants with social anxiety surprise differential electrophysiological response to vocal and music stimuli for musicians and non-

musicians

in musicians, subcortical activation is enhanced

during complex portion of the sound and

decreased during more periodic portion

Test: VER, pre/post-design 
Table 1: Summary of empirical articles on musicality and vocal emotion perception that met the selection criteria continued

\begin{tabular}{|c|c|c|c|c|c|}
\hline Reference & Sample (Mean Age) & Inclusion Criteria & Stimuli and Task & Emotions & Results \\
\hline Mualem et al., 2015 & $\begin{array}{l}\text { Exp 1: } 12 \text { intervention } \\
\text { group ( } 24 \text { y) } 12 \text { control } \\
\text { group ( } 25 \text { y); Exp 2: } 23 \\
\text { musicians ( } 26 \text { y) }\end{array}$ & $\begin{array}{l}>6 \text { years of formal music } \\
\text { training }\end{array}$ & $\begin{array}{l}\text { Intervention: } 4 \text { x } 30 \text { min music } \\
\text { sessions, control: art session, } \\
\text { focused on emotion expression; } \\
\text { Test: VER, pre/post-design }\end{array}$ & $\begin{array}{l}\text { happiness, anger, } \\
\text { fear, sadness, and } \\
\text { neutral }\end{array}$ & $\begin{array}{l}\text { Exp1: intervention group better than control } \\
\text { group in VER, Exp2: no difference between } \\
\text { musicians and non-musicians }\end{array}$ \\
\hline Nashkoff 2007 & $\begin{array}{l}42 \text { intervention group, } \\
39 \text { controls }\end{array}$ & - & $\begin{array}{l}\text { Intervention: Pitch discrimination } \\
\text { practice ( } 8 \text { weeks), Test: VER, } \\
\text { pre/post-design }\end{array}$ & $\begin{array}{l}\text { happiness, fear, } \\
\text { sadness, and } \\
\text { ambiguous }\end{array}$ & VER improved after intervention \\
\hline Cochlear implants & & & & & \\
\hline Chari et al., 2020 & $\begin{array}{l}18 \mathrm{Cl} \text { users (62 y), } \\
\text { postlingually deafened }\end{array}$ & - & $\begin{array}{l}\text { Intervention: auditory-motor, } \\
\text { auditory-only, or no training ( } 3 \\
\text { months); Test: speech, music and } \\
\text { VER tests, pre/post-design }\end{array}$ & $\begin{array}{l}\text { happiness, anger, } \\
\text { fear, sadness and } \\
\text { neutral }\end{array}$ & $\begin{array}{l}\text { training effects only in melodic contour } \\
\text { identification task }\end{array}$ \\
\hline Fuller et al., 2018 & $\begin{array}{l}19 \mathrm{Cl} \text { users (69 y), } \\
\text { postlingually deafened }\end{array}$ & - & $\begin{array}{l}\text { Intervention: music-therapy, } \\
\text { pitch/timbre group, or control (6 } \\
\text { weeks); Test: speech, music and } \\
\text { VER tests, pre/post-design }\end{array}$ & $\begin{array}{l}\text { joy, anger, sadness, } \\
\text { and relief }\end{array}$ & $\begin{array}{l}\text { Improvement of VER in music-therapy group, } \\
\text { improvement of melodic contour identification in } \\
\text { pitch/timbre group }\end{array}$ \\
\hline
\end{tabular}


Table 1: Summary of empirical articles on musicality and vocal emotion perception that met the selection criteria continued

\begin{tabular}{|c|c|c|c|c|c|}
\hline Reference & Sample (Mean Age) & Inclusion Criteria & Stimuli and Task & Emotions & Results \\
\hline Good et al., 2017 & $\begin{array}{l}18 \mathrm{Cl} \text { users, children (10 } \\
\text { y) }\end{array}$ & - & $\begin{array}{l}\text { Intervention: music (piano) or art } \\
\text { training (painting), } 6 \text { months; } \\
\text { Test: music and VER tests; pre-, } \\
\text { mid-, post-design }\end{array}$ & $\begin{array}{l}\text { happiness, anger, } \\
\text { fear, and sadness }\end{array}$ & $\begin{array}{l}\text { music perception and emotional speech prosody } \\
\text { perception improved in the music group } \\
\text { compared to the art group }\end{array}$ \\
\hline Petersen et al., & $18 \mathrm{Cl}$ users $(53 \mathrm{y}), 6 \mathrm{NH}$ & - & Intervention: 6 month one-to- & happiness and & in music group; earlier onset in improvement of \\
\hline 2012 & controls (54 y) & & $\begin{array}{l}\text { one musical ear-training, or } \\
\text { control (no task); Test: speech, } \\
\text { music and VER tests; pre-, mid-, } \\
\text { post-design }\end{array}$ & sadness & $\begin{array}{l}\text { emotional prosody perception; both } \mathrm{Cl} \text { groups still } \\
\text { worse than } \mathrm{NH} \text { controls }\end{array}$ \\
\hline Waaramaa et al., & $25 \mathrm{Cl}$ users $(12 \mathrm{y})$ and & - & vocal emotion identification task, & anger, fear, & $\mathrm{NH}$ controls performed better than $\mathrm{Cl}$ users, \\
\hline 2018 & $18 \mathrm{NH}$ controls (12 y) & & $\begin{array}{l}\text { self-reported musical interests } \\
\text { and measuremts of acoustic } \\
\text { parameters of the stimuli }\end{array}$ & $\begin{array}{l}\text { excitement, and } \\
\text { contentment }\end{array}$ & $\begin{array}{l}\text { musical interests and voice quality parameters } \\
\text { related to correct identification in both groups }\end{array}$ \\
\hline Congenital Amusia & & Amusia & & & \\
\hline Cheung et al., 2020 & $\begin{array}{l}20 \text { participants with } \\
\text { amusia ( } 22 \text { y), } 17 \\
\text { controls ( } 22 \text { y), all } \\
\text { Cantonese speakers }\end{array}$ & global MBEA score $<71 \%$ & $\begin{array}{l}\text { emotion prosody rating task, } \\
\text { emotion judgment task of } \\
\text { written words, valence judgment } \\
\text { task of written words }\end{array}$ & $\begin{array}{l}\text { happiness, anger, } \\
\text { fear, and sadness }\end{array}$ & $\begin{array}{l}\text { participants with amusia performed worse in VER } \\
\text { task than controls, no difference in tasks with } \\
\text { written words }\end{array}$ \\
\hline
\end{tabular}


Table 1: Summary of empirical articles on musicality and vocal emotion perception that met the selection criteria continued

\begin{tabular}{|c|c|c|c|c|c|}
\hline Reference & Sample (Mean Age) & Inclusion Criteria & Stimuli and Task & Emotions & Results \\
\hline Lima et al., 2016 & $\begin{array}{l}13 \text { participants with } \\
\text { amusia ( } 58 \text { y), } 11 \\
\text { controls ( } 53 \text { y) }\end{array}$ & $\begin{array}{l}\text { pitch-based MBEA scores } \\
\text { more than } 2 \text { SD below } \\
\text { population average ( } \\
72 \%)\end{array}$ & $\begin{array}{l}\text { Exp 1: rating of emotions in } \\
\text { voices and faces, Exp 2: ratings of } \\
\text { spontaneous and posed laughs }\end{array}$ & $\begin{array}{l}\text { anger, fear, } \\
\text { sadness, disgust, } \\
\text { relief, amusement, } \\
\text { and pleasure; } \\
\text { laughs }\end{array}$ & $\begin{array}{l}\text { Exp 1: impairments in amusia for all stimulus } \\
\text { types; Exp 2: participants with amusia show } \\
\text { decreased sensitivy to authenticity of laughs }\end{array}$ \\
\hline Lolli et al., 2015 & $\begin{array}{l}\text { Exp 1: } 40 \text { (aged 18-22), } \\
9 \text { considered amusic; } \\
\text { Exp 2: } 29 \text { (aged 18-28), } \\
3 \text { considered amusic }\end{array}$ & $\begin{array}{l}\text { pitch threshold }>16 \mathrm{~Hz} \text { in } \\
\text { pitch discrimination task }\end{array}$ & $\begin{array}{l}\text { Exp1: VER, low-pass filtered } \\
(500 \mathrm{~Hz}) \text { and unfiltered speech; } \\
\text { Exp 2: high-pass filtered }(4800 \mathrm{~Hz}) \\
\text { and unfiltered speech }\end{array}$ & $\begin{array}{l}\text { happiness, fear, } \\
\text { sadness, irritation, } \\
\text { tenderness and } \\
\text { neutral }\end{array}$ & $\begin{array}{l}\text { Exp 1: impairments in amusia in the low-pass } \\
\text { filtered but not in the unfiltered condition; Exp 2: } \\
\text { no differences }\end{array}$ \\
\hline Pralus et al., 2019 & $\begin{array}{l}18 \text { participants with } \\
\text { amusia ( } 33 \text { y), } 18 \\
\text { controls ( } 35 \text { y) }\end{array}$ & $\begin{array}{l}\text { global MBEA score }< \\
23 / 30(76 \%) \text { and/or a } \\
\text { MBEA pitch score }<22 / 30 \\
(73 \%)\end{array}$ & $\begin{array}{l}\text { emotion categorization and } \\
\text { intensity rating of emotional } \\
\text { sentences and vowels }\end{array}$ & $\begin{array}{l}\text { joy, anger, fear, } \\
\text { sadnes, and neutral }\end{array}$ & $\begin{array}{l}\text { worse performance of participants with amusia on } \\
\text { vowels but not sentences; intensity ratings: no } \\
\text { differences }\end{array}$ \\
\hline $\begin{array}{l}\text { Thompson et al., } \\
2012\end{array}$ & $\begin{array}{l}12 \text { participants with } \\
\text { amusia (50 y), } 12\end{array}$ & $\begin{array}{l}\text { MBEA scale subtest score }< \\
22 / 30(73 \%) \text { on two }\end{array}$ & $\begin{array}{l}\text { VER; self-report questionnaire on } \\
\text { emotional prosody perception in }\end{array}$ & $\begin{array}{l}\text { happiness, fear, } \\
\text { sadness, }\end{array}$ & $\begin{array}{l}\text { VER: participants with amusia worse than } \\
\text { controls; report awareness of VER problems in }\end{array}$ \\
\hline & controls (46 y) & consecutive occasions & daily life & $\begin{array}{l}\text { tenderness, } \\
\text { irritation, and } \\
\text { neutral }\end{array}$ & daily life \\
\hline
\end{tabular}


Table 1: Summary of empirical articles on musicality and vocal emotion perception that met the selection criteria continued

\begin{tabular}{|c|c|c|c|c|c|}
\hline Reference & Sample (Mean Age) & Inclusion Criteria & Stimuli and Task & Emotions & Results \\
\hline Zhang et al., 2018 & $\begin{array}{l}19 \text { participants with } \\
\text { amusia ( } 23 \text { y), } 19 \\
\text { controls ( } 23 \text { y), all } \\
\text { Mandarin speakers }\end{array}$ & $\begin{array}{l}\text { pitch-based MBEA score < } \\
65 / 90(72 \%)\end{array}$ & $\begin{array}{l}\text { Vocal emotion recognition, } \\
\text { speech and non-speech (low-pass } \\
\text { filtered at } 500 \mathrm{~Hz} \text { ) condition }\end{array}$ & $\begin{array}{l}\text { happiness, anger, } \\
\text { fear, sadness, } \\
\text { surprise, and } \\
\text { neutral }\end{array}$ & $\begin{array}{l}\text { performance of participants with amusia worse } \\
\text { than controls, for all emotions and conditions }\end{array}$ \\
\hline Individual differenc & & & & & \\
\hline Dibben et al., 2018 & $52(32 y)$ & - & $\begin{array}{l}\text { ratings of perceived emotion in } \\
\text { music and speech; } \\
\text { questionnaires on personality, } \\
\text { emotional intelligence and } \\
\text { musical training }\end{array}$ & valence and arousal & $\begin{array}{l}\text { ratings associated with emotional stability, } \\
\text { agreeableness, musical training (but for musical } \\
\text { stimuliy only) and age }\end{array}$ \\
\hline Globerson et al., & Exp 1: 60 (25 y); Exp 2: & - & Exp 1, Exp2: prosody recognition & happiness, anger, & psychoacoustic thresholds explained $31 \%$ and $38 \%$ \\
\hline 2013 & $37(25 y)$ & & $\begin{array}{l}\text { tasks (pragmatic and emotional) } \\
\text { and different psychoacoustic } \\
\text { tasks }\end{array}$ & fear, and sadness & $\begin{array}{l}\text { of affective and pragmatic prosody recognition } \\
\text { performance }\end{array}$ \\
\hline Trimmer \& Cuddy, & $100(22$ y) & - & VER of spech utterances and & joy, anger, fear, & emotional intelligence, not musical training \\
\hline 2008 & & & $\begin{array}{l}\text { melodic analogues; tests on } \\
\text { intelligence, emotional } \\
\text { intelligence and musical abilities }\end{array}$ & sadness and neutral & predicted vocal emotion recognition performance \\
\hline
\end{tabular}

Note: $V E R=$ vocal emotion recognition, MBEA = Montreal Battery of Evaluation of Amusia (Peretz et al., 2003) 
Table 1: Summary of empirical articles on musicality and vocal emotion perception that met the selection criteria continued

\begin{tabular}{|c|c|c|c|c|c|}
\hline Reference & Sample (Mean Age) & Inclusion Criteria & Stimuli and Task & Emotions & Results \\
\hline Waaramaa \& Leisiö, & 250; 50 per country & - & vocal emotion recognition task & happiness, anger, & emotion recognition performance above chance \\
\hline \multirow[t]{3}{*}{2013} & (Finland 48 y, Russia 35 & & with replay-option; questionnaire & fear, sadness, & in all countries, musical interests tended to have a \\
\hline & y, Estonia 32 y, Sweden & & on musical interests & surprise, disgust, & positive effect on VER \\
\hline & $27 \mathrm{y}$, and the USA $23 \mathrm{y}$ ) & & & interest and neutral & \\
\hline
\end{tabular}

